\title{
Alte, multimorbide Patienten sind besonders gefährdet
}

\author{
An den Versorgungsdaten lässt sich ablesen, dass den 80- bis 85- \\ jährigen Patienten die meisten Medikamente verordnet werden. \\ Entsprechend häufiger als bei jungen Menschen treten in dieser \\ Altersgruppe unerwünschte Arzneimittelwirkungen auf. Welche \\ Substanzen sind besonders gefährlich für ältere Menschen, und wie \\ lassen sich schwerwiegende unerwünschte Arzneimittelwirkungen \\ verhindern?
}

— Jeder Dritte über 70-Jährige nimmt täglich zwischen fünf und acht, jeder Siebte sogar mehr als 13 Wirkstoffe ein. Angesichts dieser Polypharmakotherapie besteht ein deutlich erhöhtes Risiko von unerwünschten Arzneimittelwirkungen. Hinzu kommen eine altersbedingt eingeschränkte Nierenfunktion, eine herabgesetzte metabolische Kapazität und eine gesteigerte Sensitivität der Zielorgane bzw. Rezeptoren oder Kanäle für Medikamente.

\section{Gefährliche Interaktionen}

Im Rahmen der Berliner Altenstudie wurden 516 Menschen mit einem durchschnittlichen Alter von 85 Jahren im Hinblick auf ihre Medikation befragt. Jeder zweite Patient nahm vier oder mehr Arzneimittel täglich ein, im Mittel waren es sechs Medikamente und ein bis zwei frei erhältliche Präparate. Pro Person ergaben sich somit ca. 55 potenzielle unerwünschte Arzneimittelwirkungen, wobei Übelkeit, Erbrechen und Diarrhöen an erster Stelle standen, gefolgt von Hypotonie, Schwindel und Benommenheit.

$\mathrm{Zu}$ den Arzneimitteln, die sehr häufig unerwünschte Nebenwirkungen auslösen, gehören Benzodiazepine, Kalziumantagonisten, Diuretika, nicht steroidale Antirheumatika, Digitalis, Glykoside und Antidiabetika. Am häufigsten werden entsprechende Nebenwirkungen bei Arzneimitteln mit geringer therapeutischer Breite wie z. B.
Digitalisglykoside, Diuretika oder Euphyllin beobachtet.

\section{Häufiger Anlass für eine stationäre Behandlung}

Seit 1997 wird vom Bundesinstitut für Arzneimittel und Medizinprodukte (BfArM) ein Modellprojekt zur Erfassung von unerwünschten Arzneimittelwirkungen, die zu einer stationären Aufnahme führen, gefördert. Dabei zeigt sich, dass Frauen generell signifikant häufiger von solchen medikamentösen Nebenwirkungen betroffen sind als Männer. Häufigste Verursacher einer stationären Behandlung sind Antidiabetika, nicht steroidale Antirheumatika, Antikoagulanzien und Herz-Kreislauf-Medikamente.

Die digitalisassoziierten stationären Aufnahmen sind überwiegend durch Interaktionen mit anderen Medikamenten bedingt, nämlich durch die gleichzeitige Gabe von Betablockern oder Kalziumantagonisten vom Verapamil-Typ. Diese Substanzen verstärken die negativ dromotrope Wirkung von Digitalis und können somit zu einer symptomatischen Bradykardie führen. Da über 90\% der beobachteten Nebenwirkungen durch Digitoxin und nicht durch Digoxin bedingt sind, dürfte die eingeschränkte Nierenfunktion nicht das Hauptproblem sein. Meist ist die empfohlene Tagesdosis im Hinblick auf das Körpergewicht zu hoch.

\section{Vorsicht Psychopharmaka}

Interaktionen sind angesichts der notwendigen Multimedikation bei alten Menschen nahezu unvermeidbar. Erschwerend kommt hinzu, dass selbst bei leitliniengerechter Pharmakotherapie Interaktionen auftreten können, z.B. durch die Kombination von Betablocker und Digitalis bei schwerer Herzinsuffizienz.

Bei Patienten in Pflegeheimen ist die Verordnung von Antipsychotika mit dem höchsten relativen Risiko einer unerwünschten Wirkung verbunden. Nach neueren Erhebungen beträgt die Inzidenz von unerwünschten Arzneimittelwirkungen 9,8/100 Heimbewohner-Monate, wobei jedes zweite Ereignis durchaus vermeidbar wäre. Gerade alte Menschen in Pflegeheimen erfordern nach der Verordnung von Psychopharmaka ein sorgfältiges klinisches Monitoring, um potenziell gefährliche Risiken abwenden zu können.

\section{Literatur beim Verfasser}

Anschrift des Verfassers:

Dr. med. Peter Stiefelhagen

DRK-Klinikum Westerwald, Krankenhaus

Hachenburg, D-57627 Hachenburg

E-Mail:pdrstiefel@aol.com

\section{Keywords}

Adverse Drug Reactions: Specific Hazards in Multimorbid Patients Pharmaceuticals - Interaction Undesirable side effect - Seniors 